\title{
On Generalized Semi-I-Open Sets
}

\author{
H. K. Tripathi ${ }^{1}$, J. K. Maira ${ }^{2}$ \\ ${ }^{1}$ Govt.Women's Polytechnic College, Jabalpur, India \\ ${ }^{2}$ Department of Mathematics and Computer Science, Rani Durgawati University, Jabalpur, India
}

\begin{abstract}
In this paper we have introduced the notion of g-semi-I-open sets in generalized ideal topological spaces and obtained its significant properties. We have also investigated the concept of the generalized closure operator $c_{g}^{*}$ and observed useful results
\end{abstract}

Keywords: Ideal topological space, Generalized ideal topological space, Local function, g-semi-open set.

\section{Introduction}

The notion of generalized topology was introduced by Csaszar [1] in 2002. Jancovic and Hamlett [4] have studied the concept of local function in Ideal topological spaces. Using the concepts of local function, Hatir and Noiri [3] have introduce the notion of semi-I-open sets. Maitra and Tripathi [6] have studied the concept of local function in generalized ideal topological spaces.

In this paper we have introduced the notion of g-semi-I-open sets and obtained its several properties. Further we have introduced $c_{g}^{*}$ operator on a subset of generalized ideal topological spaces and obtained significant results.

\section{Preliminaries}

First we recall definition of generalized topological space.

Definition 2.1 [1]: Let $\mathrm{X}$ be a non-empty set and let $\tau_{g}$ be a family of subsets of $\mathrm{X}$. Than $\tau_{g}$ is said to be generalized topology on $\mathrm{X}$ if following two conditions are satisfied viz;;

(i) $\emptyset, X \in \tau_{g}$,

(ii) If $G_{\lambda} \in \tau_{g}$ for $\lambda \in \Lambda$ then $\cup_{\lambda \in \Lambda} G_{\lambda} \in \tau_{g}$.

The pair $\left(X, \tau_{g}\right)$ is called generalized topological space. The elements of family $\tau_{g}$ are called g-open sets and their complements are called g-closed sets.

Example 2.1: Let us consider set $X=\left\{x_{1}, x_{2}, x_{3}\right\}$. Then we see that $\tau_{g}=\left\{\varnothing, X,\left\{x_{1}, x_{2}\right\}\left\{x_{2}, x_{3}\right\}\right\}$ is a generalized topology on $X$.

Proposition 2.1: Let $\left(X, \tau_{g}\right)$ be a generalized topological space. Then the following conditions are satisfied:

(i) $\phi$ and $X$ are g-closed sets in $\mathrm{X}$.

(ii) Arbitrary intersection of g-closed sets is a g-closed set in $X$.

Remark: We note that union of two g-closed sets in X may not be a g-closed set in $\mathrm{X}$.

Definition 2.2 [1]: Let $X$ be a generalized topological space and $A \subseteq X$. Then the g-closure of $\mathrm{A}$ is defined as the intersection of all g-closed sets in $X$ containing $A$. The gclosure of $A$ is denoted by $c_{g}(A)$.
Remark: In a generalized topological space $X$ we note that a set $A$ is g-closed iff $c_{g}(A)=A$. Further we note that $c_{g}(A)$ is the smallest $g$-closed set in $X$ containing $A$.

Proposition 2.2: Let $X$ be a generalized topological space and let $\left\{A_{\propto}\right\}_{\propto \in \Lambda}$ be a family of subsets of $X$. Then

(i) $\mathrm{U}_{\propto \in \Lambda} c_{g}\left(A_{\propto}\right) \subseteq c_{g}\left(\mathrm{U}_{\propto \in \Lambda} A_{\propto}\right)$, and

(ii) $c_{g}\left(\bigcap_{\propto \in \Lambda} A_{\propto}\right) \subseteq \bigcap_{\propto \in \Lambda} c_{g}\left(A_{\propto}\right)$.

Definition 2.3 [1]: Let $X$ be a generalized topological space and $A \subseteq X$. Then the g-interior of $A$ is defined as the union of all g-open sets in X contained in A. The g-interior of $A$ is denoted by $\boldsymbol{i}_{\boldsymbol{g}}(\boldsymbol{A})$.

Remark: In a generalized topological space $X$ we note that a set $A$ is g-open iff $i_{g}(A)=A$. Further we note that $i_{g}(A)$ is the largest g-open set in $X$ contained in $A$.

Proposition 2.3: Let $X$ be a generalized topological space and let $\left\{A_{\propto}\right\}_{\propto \in \Lambda}$ be a family of subsets of $X$. Then

(i) $\mathrm{U}_{\propto \in \Lambda} i_{g}\left(A_{\propto}\right) \subseteq i_{g}\left(\mathrm{U}_{\propto \in \Lambda} A_{\propto}\right)$, and

(ii) $i_{g}\left(\bigcap_{\propto \in \Lambda} A_{\propto}\right) \subseteq \bigcap_{\propto \in \Lambda} i_{g}\left(A_{\propto}\right)$.

Proposition 2.4: Let $X$ be a generalized topological space and $A \subseteq X$. Then

(i) $i_{g}(X-A)=X-c_{g}(A)$, and

(ii) $c_{g}(X-A)=X-i_{g}(A)$.

\section{Generalized Closure Operator on Subsets of $\mathbf{X}$}

In this section we have introduced the notion of $c_{g}^{*}$, the generalized closure operator on the family of all subsets of $X$, where $\mathrm{X}$ is a generalized topological space. We have obtained significant results of the $c_{g}^{*}$ operator. We begin with the definition of Ideal on a generalized topological space.

Definition 3.1 [6]: Let $\left(X, \tau_{g}\right)$ be a generalized topological space and let $I$ be a family of subsets of $X$. Then $I$ is said to be an Ideal on $X$ if following two conditions are satisfied viz;

(i) Hereditary property:- If $A \in I$ and $B \subseteq A$ then $B \in I$.

(ii) Finite additivity:- If $A, B \in I$ then $A \cup B \in I$.

The triplet $\left(X, \tau_{g}, I\right)$ is called generalized ideal topological space. 


\section{International Journal of Science and Research (IJSR) \\ ISSN (Online): 2319-7064 \\ Index Copernicus Value (2013): 6.14 | Impact Factor (2014): 5.611}

Example 3.1: Let us consider set $X=\left\{x_{1}, x_{2}, x_{3}\right\}$ and let $\tau_{g}=\left\{\varnothing, X,\left\{x_{1}, x_{2}\right\},\left\{x_{2}, x_{3}\right\}\right\}$ be generalized topology on $X$. Let $I=\left\{\varnothing,\left\{x_{1}\right\},\left\{x_{2}\right\},\left\{x_{1}, x_{2}\right\}\right\}$. Then $I$ is an ideal on the generalized topological space $X$, and $\left(X, \tau_{g}, I\right)$ is a generalized ideal topological space.

The collection of Ideals on a generalized topological space X is closed under arbitrary intersection operation, viz.:

Proposition 3.1: Let $X$ be a generalized topological space and let $\left\{I_{\lambda}\right\}_{\lambda \in \Lambda}$, where $\Lambda$ is an index set, be any arbitrary collection of ideals on $\mathrm{X}$. Then $I=\bigcap_{\lambda \in \Lambda} I_{\lambda}$ is an ideal on $\mathrm{X}$.

Proof: Suppose $A \in I$ and $B \subseteq A$. Then $A \in I_{\lambda}$ for each $\lambda \in \Lambda$. Since $I_{\lambda}$ is an ideal on $\mathrm{X}$ and $B \subseteq A$, we have $\mathrm{B} \in I_{\lambda}$. Therefore $\mathrm{B} \in I_{\lambda}$ for each $\lambda \in \wedge$. Hence $\mathrm{B} \in \bigcap_{\lambda \in \Lambda} I_{\lambda}=I$. Further suppose that $A_{1}, A_{2} \in I$. This implies $A_{1}, A_{2} \in I_{\lambda}$ for each $\lambda \in \wedge$. As $I_{\lambda}$ is an ideal on $\mathrm{X}$, we have $A_{1} \cup A_{2} \in I_{\lambda}$ for each $\lambda \in \wedge$. Hence $A_{1} \cup A_{2} \in \underset{\lambda \in \Lambda}{\cap} I_{\lambda}=I$. Thus $I=\bigcap_{\lambda \in \Lambda} I_{\lambda}$ is an ideal on $\mathrm{X}$.

However, collection of ideals on a generalized topological space $X$ is not closed under the operation of union. We have following Example.

Example 3.2: Let us consider generalized topological space $X=\left\{x_{1}, x_{2}, x_{3}\right\}$ with generalized topology $\tau_{g}=\{\varnothing, X$, $\left.\left\{x_{1}, x_{2}\right\},\left\{x_{1}, x_{3}\right\}\right\}$. Further let us consider ideals $I_{1}=$ $\left\{\varnothing,\left\{x_{1}\right\}\left\{x_{2}\right\},\left\{x_{1}, x_{2}\right\}\right\}$ and $I_{2}=\left\{\varnothing,\left\{x_{1}\right\}\left\{x_{3}\right\},\left\{x_{1}, x_{3}\right\}\right\}$ on $\mathrm{X}$. Then we can see that $I_{1} \cup I_{2}=\left\{\varnothing,\left\{x_{1}\right\},\left\{x_{2}\right\}\right.$, $\left.\left\{x_{3}\right\},\left\{x_{1}, x_{2}\right\},\left\{x_{1}, x_{3}\right\}\right\}$ is not an ideal on $X$.

Definition 3.2: Let $\left(X, \tau_{g}, I\right)$ be a generalized ideal topological space, and let $\mathrm{A} \subseteq \mathrm{X}$. Then the local function of A is denoted by $A^{*}$ and is defined as $A^{*}=\{x \in X: A \cap \cup \notin$ Ifor each $g-$ open set Ucontaining $x$.

Definition 3.3: Let $\left(X, \tau_{g}, I\right)$ be a generalized ideal topological space. Then the operator $c_{g}^{*}: \mathrm{P}(\mathrm{X}) \rightarrow \mathrm{P}(\mathrm{X})$, where $\mathrm{P}(\mathrm{X})$ is a power set of $\mathrm{X}$, defined as $c_{g}^{*}(A)=A \cup A^{*}$ for all $A \in \mathrm{P}(\mathrm{X})$ is called generalized closure operator.

Example 3.3: Let $X=\left\{x_{1}, x_{2}, x_{3}\right\}$ be a generalized topological space with generalized topology $\tau_{g}=$ $\left\{\varnothing, X,\left\{x_{1}, x_{2}\right\},\left\{x_{2}, x_{3}\right\}\right\}$ and ideal $I=\left\{\varnothing,\left\{x_{1}\right\}\right\}$ on $\mathrm{X}$. Then generalized closure operator an each subsets of $\mathrm{X}$ is defined as follows:

- $c_{g}^{*}(\varnothing)=\emptyset$.

- $c_{g}^{*}\left\{x_{1}\right\}=\left\{x_{1}\right\}$.

- $c_{g}^{*}\left\{x_{2}\right\}=X$.

- $c_{g}^{*}\left\{x_{3}\right\}=\left\{x_{3}\right\}$.

- $c_{g}^{*}\left\{x_{1}, x_{2}\right\}=X$.

- $c_{g}^{*}\left\{x_{1}, x_{3}\right\}=\left\{x_{1}, x_{3}\right\}$.

- $c_{g}^{*}\left\{x_{2}, x_{3}\right\}=X$.

- $c_{g}^{*}(X)=X$.
Proposition 3.2: Let $X$ be a generalized ideal topological space and $A \subseteq X$. Then $c_{g}^{*}(A) \subseteq c_{g}(A)$.

Proof: Let $X$ be a generalized ideal topological space and $A \subseteq X$. Let $x \in A^{*}$ and $\mathrm{F}$ be a g-closed set such that $A \subseteq F$. Then $X-F$ is a g-open set and it is disjoint from $A$. This implies $x \notin X-F$. i.e., $x \in F$. Hence $A^{*} \subseteq F$ and we have, $A^{*} \subseteq c_{g}(A)$. Since $c_{g}^{*}(A)=A \cup A^{*} \subseteq A \cup c_{g}(A)$, it follows that $c_{g}^{*}(A) \subseteq c_{g}(A)$.

Proposition 3.3: Let $X$ be a generalized ideal topological space and let A, B be subsets of $X$. Then

(i) $\quad c_{g}^{*}(\phi)=\phi, c_{g}^{*}(X)=X$.

(ii) If $A \subseteq B$ then $c_{g}^{*}(A) \subseteq c_{g}^{*}(B)$.

(iii) $\quad c_{g}^{*}(A) \cup c_{g}^{*}(B) \subseteq c_{g}^{*}(A \cup B)$.

(iv) $\quad c_{g}^{*}(A \cap B) \subseteq c_{g}^{*}(A) \cap c_{g}^{*}(B)$.

Proof: (i) Since $\quad c_{g}^{*}(\phi)=\phi \cup \phi^{*}=\phi \cup \phi=\phi$. Thus $c_{g}^{*}(\phi)=\phi$.

$$
\text { Now } c_{g}^{*}(X)=X \cup X^{*}=X \text {. }
$$

(ii) Let $X$ be a generalized ideal topological space and $A \subseteq B \subseteq X$. Then we have, $A^{*} \subseteq B^{*}$. Now $c_{g}^{*}(A)=A \cup$ $A^{*} \subseteq B \cup B^{*}=c_{g}^{*}(B)$. Thus $c_{g}^{*}(A) \subseteq c_{g}^{*}(B)$.

(iii) Since $A \subseteq A \cup B, B \subseteq A \cup B$ from (ii) we have $c_{g}^{*}(A) \subseteq c_{g}^{*}(A \cup B)$ and $c_{g}^{*}(B) \subseteq c_{g}^{*}(A \cup B)$. This implies $c_{g}^{*}(A) \cup c_{g}^{*}(B) \subseteq c_{g}^{*}(A \cup B)$.

(iv) Since $A \cap B \subseteq A$ and $A \cap B \subseteq B$ from (ii) we have $c_{g}^{*}(A \cap B) \subseteq c_{g}^{*}(A)$ and $c_{g}^{*}(A \cap B) \subseteq c_{g}^{*}(B)$. This implies $c_{g}^{*}(A \cap B) \subseteq c_{g}^{*}(A) \cap c_{g}^{*}(B)$.

Proposition 3.4: Let $X$ be a generalized ideal topological space and let $\left\{A_{\propto}\right\}_{\propto \in \Lambda}$ be a family of subsets of $X$. Then

(i) $\mathrm{U}_{\propto \in \Lambda} c_{g}^{*}\left(A_{\propto}\right) \subseteq c_{g}^{*}\left(\mathrm{U}_{\propto \in \Lambda} A_{\propto}\right)$, and

(ii) $\quad c_{g}^{*}\left(\bigcap_{\propto \in \Lambda} A_{\propto}\right) \subseteq \bigcap_{\propto \in \Lambda} c_{g}^{*}\left(A_{\propto}\right)$.

Proof: (i) Let $\left\{A_{\propto}\right\}_{\propto \in \Lambda}$ be a collection of subsets of generalized topological space $X$. Then we have $A_{\propto} \subseteq$ $\bigcup_{\alpha \in \Lambda} A_{\propto}$, for each $\propto \in \Lambda$. This implies $c_{g}^{*}\left(A_{\propto}\right) \subseteq$ $c_{g}^{*}\left(\mathrm{U}_{\propto \in \Lambda} A_{\propto}\right)$, for each $\propto \in \Lambda$. Therefore $\mathrm{U}_{\propto \in \Lambda} c_{g}^{*}\left(A_{\propto}\right) \subseteq$ $c_{g}^{*}\left(\mathrm{U}_{\propto \in \Lambda} A_{\propto}\right)$.

(ii) We have $\bigcap_{\propto \in \Lambda} A_{\propto} \subseteq A_{\propto}$, for each $\propto \in \Lambda$. This implies $c_{g}^{*}\left(\bigcap_{\propto \in \Lambda} A_{\propto}\right) \subseteq c_{g}^{*}\left(A_{\propto}\right)$, for each $\propto \in \Lambda$. Hence we conclude that $c_{g}^{*}\left(\bigcap_{\propto \in \Lambda} A_{\propto}\right) \subseteq \bigcap_{\propto \in \Lambda} c_{g}^{*}\left(A_{\propto}\right)$.

\section{4. g-semi-I- open sets}

In this section, we have studied the notion of g-semi-I- open sets in a generalized ideal topological space and observed its properties. First we recall the definition of g-semi- open set.

Definition 4.1[2]: Let $\left(X, \tau_{g}\right)$ be a generalized topological space and $\mathrm{A} \subseteq \mathrm{X}$. Then, $\mathrm{A}$ is said to be g-semi-open set if $A \subseteq c_{g}\left(i_{g}(A)\right)$.

Proposition 4.1: In a generalized topological space each gopen set is g-semi-open. 


\section{International Journal of Science and Research (IJSR) \\ ISSN (Online): 2319-7064 \\ Index Copernicus Value (2013): 6.14 | Impact Factor (2014): 5.611}

Proof: $\mathrm{X}$ be a generalized topological space and let $\mathrm{A}$ be a gopen set in $\mathrm{X}$. Then $A=i_{g}(A)$. Since $\mathrm{A} \subseteq c_{g}(A)$, we have $A \subseteq c_{g}\left(i_{g}(A)\right)$. Hence $\mathrm{A}$ is a g-semi-open set in $\mathrm{X}$.

The converse of above result is not necessarily true. We have following example:

Example 4.1: Let us consider set $X=\left\{x_{1}, x_{2}, x_{3}, x_{4}\right\}$ with generalized topology $\tau_{g}=\left\{\phi, X,\left\{x_{1}, x_{2}\right\},\left\{x_{2}, x_{3}\right\},\left\{x_{1}, x_{2}, x_{3}\right\}\right.$ on $\mathrm{X}$. Then the family of g-closed sets is given by $\tau_{g}^{c}=\left\{\phi, X,\left\{x_{4}\right\},\left\{x_{3}, x_{4}\right\}\right.$, $x 1, x 4$. Suppose $A=x 1, x 2, x 4$. Then $i g A=\{x 1, x 2\}$. Now $\operatorname{cg}(i g A=\operatorname{cg} x 1, x 2=X$. Therefore $A \subseteq \operatorname{cg}(i g(A))$. Hence A is a g-semi-open set in $\mathrm{X}$, but $A$ is not g-open set in $X$.

Definition 4.2: Let $\left(X, \tau_{g}, I\right)$ be a generalized ideal topological space and $\mathrm{A} \subseteq \mathrm{X}$. Then $\mathrm{A}$ is said to be $\mathbf{g}$-semi-Iopen set if $A \subseteq c_{g}^{*}\left(i_{g}(A)\right)$.

Proposition 4.2: In a generalized ideal topological space $\left(X, \tau_{g}, I\right)$ each g-open set is g-semi-I-open.

Proof: Let $\left(X, \tau_{g}, I\right)$ be a generalized ideal topological space and let $\mathrm{A}$ be a g-open set in $\mathrm{X}$. Then $A=i_{g}(A)$. Now $c_{g}^{*}\left(i_{g}(A)\right)=c_{g}^{*}(A)=A \cup A^{*} \supseteq A$. Thus we have $A \subseteq$ $c_{g}^{*}\left(i_{g}(A)\right)$. Hence $\mathrm{A}$ is g-semi-I-open set in $\mathrm{X}$.

The converse of above result is not necessarily true. We have following example:

Example 4.2: Let us consider set $X=\left\{x_{1}, x_{2}, x_{3}, x_{4}\right\}$ with generalized topology $\tau_{g}=\left\{\phi, X,\left\{x_{1}, x_{2}\right\},\left\{x_{2}, x_{3}\right\},\left\{x_{1}, x_{2}, x_{3}\right\}\right.$ and $I=\left\{\phi,\left\{x_{3}\right\}\right\}$ on $X$. Let $A=\left\{x_{2}, x_{3}, x_{4}\right\}$. Then $i_{g}(A)=\left\{x_{2}, x_{3}\right\}$. Now $c_{g}^{*}\left(i_{g}(A)=c_{g}^{*}\left(\left\{x_{2}, x_{3}\right\}\right)=\left\{x_{2}, x_{3}\right\} \cup\left\{x_{2}, x_{3}\right\}^{*}=\right.$

$\left\{x_{2}, x_{3}\right\} \cup X=X$. Therefore $A \subseteq c_{g}^{*}\left(i_{g}(A)\right)$. Hence A is a $g$ semi-I-open set in X but $A$ is not g-open set in $X$.

Proposition 4.3: In a generalized ideal topological space each $\mathrm{g}$-semi-I -open set is $\mathrm{g}$-semi-open.

Proof: Let $\left(X, \tau_{g}, I\right)$ be a generalized ideal topological space let $\mathrm{A}$ be a g-semi-I-open set in $\mathrm{X}$. Then we have $A \subseteq$ $c_{g}^{*}\left(i_{g}(A)\right)$. Since $c_{g}^{*}\left(i_{g}(A)\right) \subseteq c_{g}\left(i_{g}(A)\right)$, it follows that $A \subseteq c_{g}\left(i_{g}(A)\right)$. Hence $\mathrm{A}$ is g-semi-open set in $\mathrm{X}$.

The converse of above result is not necessarily true. We have following example:

Example 4.3: Let us consider set $X=\left\{x_{1}, x_{2}, x_{3}, x_{4}\right\}$ with generalized topology $\tau_{g}=\left\{\varnothing, X,\left\{x_{1}\right\},\left\{x_{1}, x_{2}\right\}\right.$, $\left.\left\{x_{2}, x_{3}\right\},\left\{x_{1}, x_{2}, x_{3}\right\}\right\}$ and ideal $I=\left\{\varnothing,\left\{x_{1}\right\}\right\}$ on $X$. Suppose $\mathrm{A}=\left\{x_{1}, x_{4}\right\}$. Then $\quad c_{g}\left(i_{g}(A)\right)=c_{g}\left(i_{g}\left\{x_{1}, x_{4}\right\}\right)=$ $c_{g}\left(\left\{x_{1}\right\}\right)=\left\{x_{1}, x_{4}\right\}$. Thus $A=c_{g}\left(i_{g}(A)\right)$. Hence $\mathrm{A}$ is $\mathrm{g}$ semi -open set in $X$. Now $c_{g}^{*}\left(i_{g}(A)\right)=c_{g}^{*}\left(i_{g}\left\{x_{1}, x_{4}\right\}\right)=c_{g}^{*}\left\{x_{1}\right\}=\left\{x_{1}\right\} \cup\left\{x_{1}\right\}^{*}=\left\{x_{1}\right\} \cup$ $\emptyset=\left\{x_{1}\right\}$. Thus $A \nsubseteq c_{g}^{*}\left(i_{g}(A)\right)$. Therefore we see that $\mathrm{A}$ is $\mathrm{g}$-semi-open but not g- semi- I-open set in X.

Proposition 4.4: In a generalized ideal topological space arbitrary union of g-semi-I-open sets is g-semi-I-open.

Proof: Let $X$ be a generalized ideal topological space and let $\left\{A_{\propto}\right\}_{\propto \in \Lambda}$ be a family of g-semi-I-open sets in X. Then $A_{\propto} \subseteq c_{g}^{*}\left(i_{g}\left(A_{\propto}\right)\right)$, for all $\propto \in \Lambda$. Suppose $A=\bigcup_{\propto \in \Lambda} A_{\propto}$. Now we have $\quad c_{g}^{*}\left(i_{g}(A)\right)=c_{g}^{*}\left(i_{g}\left(\mathrm{U}_{\propto \in \Lambda} A_{\propto}\right)\right) \supseteq$ $c_{g}^{*}\left(\mathrm{U}_{\propto \in \Lambda}\left(i_{g}\left(A_{\propto}\right)\right)\right) \supseteq \mathrm{U}_{\propto \in \Lambda}\left(c_{g}^{*}\left(\left(i_{g}\left(A_{\propto}\right)\right) \supseteq \mathrm{U}_{\propto \in \Lambda} A_{\propto}=A\right.\right.$. Thus $A=\mathrm{U}_{\propto \in \Lambda} A_{\propto}$ is a g-semi-I-open sets in X.

However, intersection of two g-semi-I-open sets is not necessarily g-semi-I-open set. We have following example:

Example 4.4: Let us consider set $X=\left\{x_{1}, x_{2}, x_{3} x_{4}\right\}$ with generalized topology $\tau_{g}=\left\{\varnothing, X,\left\{x_{1}, x_{2}\right\},\left\{x_{2}, x_{3}\right\},\left\{x_{1}, x_{2}, x_{3}\right\}\right\}$ and ideal $I=\left\{\varnothing,\left\{x_{3}\right\}\right\}$ on $X$. Suppose $\mathrm{A}=\left\{x_{1}, x_{2}, x_{4}\right\}$ and $\mathrm{B}=\left\{x_{2}, x_{3}, x_{4}\right\}$. Then we see that the sets $\mathrm{A}$ and $\mathrm{B}$ are $\mathrm{g}$ semi-I-open sets in X, but $A \cap B=\left\{x_{2}, x_{4}\right\}$ is not g-semi-Iopen set in $\mathrm{X}$.

\section{References}

[1] Csaszar; A; Generalized topology, generalized continuity, Acta Math. Hungar., 96 (2002), 351-357.

[2] Csaszar, A., Generalized open sets in generalized topologies. Acta Mathematica Hangarica, 2005, 106, 53 66.

[3] Hatir E. (Konya) and Noiri T. (Yatsushiro), On Semi-IOpen sets and Semi-I-Continuous functions, Acta. Math. Hungar. 107(4) (2005), 345-353.

[4] Jankovic D and Hamlett T.R., New Topologies from old via Ideals, The American Mathematical Monthly. Vol.97. No. 4 (Apr. 1990), pp. 295-310.

[5] Kuralowski, K. Topologic, Vol. 1 Wars zawa (1930).

[6] Maitra J.K. and Tripathi H.K., (2014), Local Function in Generalized ideal topological spaces, VISLESANA, 11(1), 191-195.

[7] Maitra J.K., Tiwari Rajesh and Tiwari V., (2015), A Note on Local Function in Generalized Ideal topological space. International Journal of Mathematics and Computer Applications and Research (IJMCAR) vol. 5, issue 5, oct,2015, 7-12.

[8] Samuels P., A Topology formed from a given topology and ideal, J. London Math. Soc., 10 (1975) 409-416. 